\title{
Prevalence of monogenic autoinflammatory diseases among Pediatric Rheumatology centers: the Eurofever PReS/PRINTO
} survey

\author{
M Gattorno*1, J Frenkel ${ }^{2}$, S Ozen ${ }^{3}$, F De Benedetti ${ }^{4}$, I Konè-Paut ${ }^{5}$, N Neven ${ }^{6}$, \\ H Girschick ${ }^{7}$, H Özdogan ${ }^{8}$, C Wouters ${ }^{9}$, P Woo ${ }^{10}$, M Hofer ${ }^{11}$, P Dolezalova ${ }^{12}$, \\ N Toplak ${ }^{13}$, V Richard ${ }^{14}$, A Martini ${ }^{1}$ and N Ruperto ${ }^{1}$
}

\begin{abstract}
Address: ${ }^{1}$ UO Pediatria II, Istituto Gaslini and Dipartimento di Pediatria, Università di Genova, Genova, Italy, ${ }^{2}$ University Medical Center, Utrecht, Utrecht, Italy, ${ }^{3}$ Hacettepe University Faculty of Medicine, Ankara, Turkey, ${ }^{4}$ Bambino Gesù Pediatric Hospital, Roma, Italy, ${ }^{5}$ Centre HospitaloUniversitaire de Bicêtre, Le Kremlin Bicêtre, France, ${ }^{6}$ Hopital Necker-Enfants Malades, Paris, France, 7 University of Würzburg, Würzburg, Germany, ${ }^{8}$ University of Istanbul, Cerrahpa\&\#351; a Medical Faculty, Istanbul, Turkey, ${ }^{9}$ Universitair Ziekenhuis Leuven, Leuven, Belgium, ${ }^{10}$ University College London, London, UK, ${ }^{11}$ Centre Hospitalier Universitaire Vaudois, Lausanne, Switzerland, ${ }^{12}$ Department of Paediatrics, Charles University, Prague, Czech Republic, ${ }^{13}$ Department of Rheumatology University Children's Hospital, Ljubljana, Slovenia and ${ }^{14}$ Faculty Children's Hospital 1 st Department for Children and Adolescents, Kosice, Slovakia

* Corresponding author
\end{abstract}

from $15^{\text {th }}$ Paediatric Rheumatology European Society (PreS) Congress

London, UK. 14-17 September 2008

Published: 15 September 2008

Pediatric Rheumatology 2008, 6(Suppl I):PI94 doi:10.I I86/I546-0096-6-SI-PI94

This abstract is available from: http://www.ped-rheum.com/content/6/SI/PI94

(c) 2008 Gattorno et al; licensee BioMed Central Ltd.

\section{Background}

Due to the rarity of Autoinflammatory diseases, affected patients are usually seen in a number of different centers. Aim of the study was to analyze the prevalence of the autoinflammatory diseases followed by Centers of Pediatric Rheumatology.

\section{Methods}

A secured web-based questionnaire on the number patients with genetically defined or clinical suspected Autoinflammatory disorders among the centers of Pediatric Rheumatology members of the Pediatric Rheumatology Trial International Organization (PRINTO, http:// www.printo.it) was performed.

\section{Results}

126 Centers from 38 Countries (94 in Europe, 13 South America, 12 Asia, 5 Australia, 2 Africa) replayed to the survey. Among the patients with a genetic proven disease 73 were affected by TRAPS, 118 by HyperIgD syndrome, 153 by cryopyrinopathies, 71 by PAPA syndrome, 66 patients by Blau's syndrome. A genetically proven FMF was reported in 2484 patients (1947 from countries of the Middle east and north Africa, 537 from other countries). A number of patients with a clinical suspected Autoinflammatory were also reported: 68 with suspected TRAPS, 84 HyperIgD, 57 cryopyrinopathies, 50 PAPA, 60 Blau's syndrome. The ratio between genetically proven vs suspected disease was 1.97 in Western European countries and 0.6 in Countries where the molecular analysis is not available.

\section{Conclusion}

A relevant number of patients with genetically defined or clinical suspected Autoinflammatory diseases are followed by different Centers of Pediatric Rheumatology worldwide. A network of registries for the proper collection of data coming from these patients and an improvement of the possibilities for the molecular diagnosis in non-Western European Countries are recommended. 